\title{
Miller Fisher-Guillain-Barré overlap syndrome during the Zika Virus outbreak
}

\author{
Wilma González-Barreto ${ }^{1}$, Gloria M. Rodríguez-Vega ${ }^{\star, 2}$, Patricia De Jesús- \\ Umpierre $^{3,4}$, José R. Rodríguez-Vázquez ${ }^{5}$
}

${ }^{1}$ Medical Sciences Campus, University of Puerto Rico, San Juan, Puerto Rico, ${ }^{2}$ Department of Critical Care Medicine, HIMA.San Pablo Hospital, Caguas, Puerto Rico, ${ }^{3}$ Multiple Sclerosis Center HIMA.San Pablo, HIMA-Ambulatory Center, Caguas, Puerto Rico, ${ }^{4}$ Department of Neurology, Medical Sciences Campus, University of Puerto Rico, San Juan, Puerto Rico, ${ }^{5}$ School of Medicine, Ponce Health Sciences University, Ponce, Puerto Rico

\begin{abstract}
Miller Fisher is a variant of Guillain-Barré syndrome, presented mainly with ataxia, areflexia and ophthalmoplegia. After the 2016 Zika virus (ZIKV) outbreak, an association between Guillain-Barré syndrome and ZIKV was established. ZIKV is a mosquito-borne-flavivirus, considered a mild disease with symptoms including fever, rash, conjunctivitis, and arthralgia. Most of cases with ZIKV infection are asymptomatic, however in neurologic complications including Guillain-Barré syndrome, symptoms could evolve leading to fatalities. A case of Miller Fisher-Guillain-Barré overlap syndrome during the 2016 ZIKV outbreak is presented.
\end{abstract}

Keywords: Miller Fisher-Guillain-Barré overlap syndrome, Miller Fisher variant, Guillain-Barré syndrome, Zika Virus infection, Puerto Rico

\section{Introduction}

Zika virus (ZIKV) is a mosquito-borneflavivirus transmitted primarily by Aedes mosquitoes, considered a mild disease with symptoms including fever, rash, conjunctivitis, and arthralgia [1]. Most of cases with ZIKV infection are asymptomatic; however an increase of congenital defects, neurologic complications, severe thrombocytopenia, and fatal cases has been reported [2, 3].

After the ZIKV outbreak in the French Polynesia in 2013, an increase in neurologic symptoms compatible with Guillain-Barré

Received: October 2017; Accepted after review: November 2017; Published: December 2017.

*Corresponding author: Gloria M. RodríguezVega, MD, FACP, FCCP, FCCM, P.O. Box 4980 Caguas, Puerto Rico 00726-4980,

E-mail: glorie68@gmail.com syndrome (GBS) were reported [3]. Similarly, on 2015 an increase in the number of cases with congenital defects and neurologic complications secondary to ZIKV were reported in the Americas [4]. GBS is considered an immune-mediated condition characterized by different degrees of weakness, sensory abnormalities, and autonomic dysfunction associated to damage of peripheral nerve or nerve root $[1,5]$. While a good prognosis has been reported, nearly $20 \%$ of patients with GBS remain disabled, including patients with overlap variants [5].

Miller Fisher (MF), a rare variant of GBS, was first described in 1956 due to its unique clinical symptoms, including ophthalmoplegia, areflexia, and ataxia [3]. Geographic differences in MF have been noted; in Western countries MF comprises $1-5 \%$ of GBS cases compared to $19 \%$ of GBS cases in Asian countries. Like GBS, MF cases are predominantly male, and incidence increases 
with age [5]. Most patients recover within several weeks or months, while $<5 \%$ are fatal cases [5, 6]. While MF has been described a unique triad of symptoms, other neurologic signs has been reported including overlapping with other variants of GBS [6].

This is the case of a 49-year-old man admitted to the Intensive Care Unit (ICU) with Miller Fisher-Guillain-Barré overlap syndrome (MF/GBS) during the 2016 ZIKV epidemic in Puerto Rico.

\section{Case Report}

On early 2016, a 49-year-old man presented to the emergency room (ER) with double vision, bilateral facial paralysis, ataxic gait, ascending weakness, and difficulty walking properly. Past medical history includes diabetes mellitus, and hyperlipidemia; he denied toxic habits, vaccines, contact with a sick person, or recent travel.

On day 0 the patient described unquantified fever, redness of eyes, and arthralgia. On day 1 of symptoms, he visited an ER where he was diagnosed with a possible viral syndrome; an antibiotic was prescribed for 5 days and was discharge home. Illness symptoms resolved within the first 4 days; however on day 4 the patient started feeling swelling and tingling sensation in feet, arms and back. He visited another ER; a pain medication was prescribed, and was discharged home. From day 5 to 6 , he developed headache, poor vision, numbness on bilateral upper and lower extremities, heaviness sensation in legs, and pain on the right side of the body. On day 6 , a facial deviation (right side) was presented. On day 8 , he went to another ER with the above symptoms; moreover he presented dysarthria. Laboratories, chest radiography, and a head computational tomography were done with normal findings; orphenadrine and opioids were ordered, and was discharge home. On day 14 , he visited an outpatient clinic and was referred to a neurologist. On day 16 patient returns to an ER with double vision, mild slurred speech, bilateral facial paralysis, ataxia, areflexia, and ascending weakness. $\mathrm{He}$ was transferred to our institution to be evaluated by a neurologist.
Upon admission, body temperature was $36.1^{\circ} \mathrm{C}$, blood pressure $143 / 104 \mathrm{mmHg}$, pulse 76 beats per minute, respiratory rate of 18 breaths per minute. On physical examination, patient was acutely ill, awake, alert, and oriented. System review was remarkable for diplopia, ataxic gait, poor coordination, and ascending weakness. On neurologic examination patient had bilateral facial weakness with a prominence in the left side as compared with right side, decreased sensation in left trigeminal nerve, including ophthalmic, maxillary, and mandibular nerves (V1-V3), and left cranial nerve $\mathrm{VI}$ paralysis. Motor strength was slightly diminished on upper extremities (5-/5), except triceps with slightly greater weakness $(4+/ 5)$; in lower extremities mild weakness (4/5) was identified in both sides, more prominent in left side (4-/5). Furthermore, patient had diminished sensation to pinprick and soft touch involving right-sided extremities, bilateral diminished vibration, and adequate proprioception. On cerebellar evaluation patient had bilateral past pointing on finger to nose test more prominent on the right side. Areflexia was detected in biceps, patellar and Achillian deep tendon reflexes bilaterally and hypoactive in brachioradialis and triceps muscles; bilateral plantar flexor responses were observed. Due to neurologic findings including cranial nerve involvement and rapid progression of symptoms, an acute inflammatory demyelinating polyneuropathy was suspected, and supportive care in the ICU was recommended.

Upon admission to the ICU, laboratories and a complete spinal cord magnetic resonance were done without abnormal findings related to the symptoms. Serum samples were analyzed for ZIKV, dengue (DENV), chikungunya, and Influenza A \& B viruses. Results were positive for ZIKV and subsequently to DENV antibodies by Zika IgM enzyme-linked immunosorbent array (ELISA). On day 16 patient was treated with a 5-days course of intravenous immunoglobulin (IVIg) $(0.4 \mathrm{~g} / \mathrm{kg} /$ day) infusion therapy.

On day 17, a lumbar puncture was performed; albuminocytologic dissociation was confirmed with total micro protein of 114.2 $\mathrm{mg} / \mathrm{dL}$; white blood cells $1 \mathrm{mg} / \mathrm{microL}$, and glucose $67.3 \mathrm{mg} / \mathrm{dL}$. From day $19-21$, the patient develops severe headache, unresolved 
with acetaminophen, opioids and gabapentin. On day 21, headache resolved and IVIg treatment was completed. By day 22, the bilateral facial weakness, reduce sensation at left mandibular nerves (V1-V3), left cranial nerve VI palsy, and diplopia persisted. Motor strength was equivocal with detectable weakness on bilateral upper extremities and left lower extremity, while definite strength with slight weakness on right lower extremity. The patient remained with diminished sensation in extremities, and absent reflexes in lower extremities; reflexes were normal in upper extremities. By day 25, the patient reported improvement of weakness, however bilateral facial paralysis, diplopia, and ptosis persisted; he was discharge home, with long-term follow up with neurology and physical therapy services.

One year after the onset of first symptoms limb weakness and ataxia resolved; reflexes were recovered, excluding biceps. On the other hand, bilateral facial paralysis and sensory changes including left $\mathrm{V} 1-\mathrm{V} 3$, diminished sensation to pinprick and soft touch (right side), and bilateral diminished vibration continued.

\section{Discussions}

Recently, GBS has been associated with antecedent infection with ZIKV [1, 7]. The incidence of GBS cases during active ZIKV in Puerto Rico is estimated to be 3.2-5.1 times the incidence during non-active ZIKV, reported as 1.7 cases per 100,000 population [8, 9]. Likewise, an increase in the incidence of GBS in 7 countries of America has been reported, with 2.0-9.8 times higher as compared with non-active ZIKV transmission [10]. In Puerto Rico, a total of 56 cases of GBS were reported from January $1^{\text {st }}-$ July $31^{\text {st }}, 2016$ of which 34 $(61 \%)$ had evidence of ZIKV or flavivirus infection [7].

Laboratory findings suggested a flavivirus infection with positive results for ZIKV IgM ELISA; however a second test of the samples revealed DENV antibodies by IgM ELISA. In cases with evidence of recent flavivirus infection, clinical and epidemiological evidence should be considered to establish an association between neurologic symptoms and
ZIKV infection [11-13]. The present case was attributed to ZIKV infection due to location of exposure, clinical signs and symptoms, and epidemiologic data reported by health authorities. During November $1^{\text {st }}-$ July $7^{\text {th }}$, 2016, most cases were attributed to ZIKV infection, as 5,582 cases were confirmed and presumptive with ZIKV infection, while 136 to DENV [7]. As in the present case, most of cases with GBS had an unspecified flavivirus test results; [7] however most of them were associated to ZIKV infection, as ZIKV was predominantly circulating compared with other flavivirus including DENV and chikungunya [12]. Due to cross reactivity of ZIKV IgM antibody with other related flaviviruses, the use of plaque reduction neutralization testing has been recommended for ZIKV identification; however is not recommended in Puerto Rico $[13,14]$.

Among GBS cases, $5 \%$ of the cases are related to the MF variant [5]. The most common clinical signs reported in patients with MF include ataxia, areflexia, and ophthalmoplegia, yet incomplete and overlapping variants could be presented. Although a good prognosis has been reported in MF, the overlap with other variants of GBS represents a life-threatening risk requiring supportive care $[15,16]$. In the present case, the patient had ophthalmoplegia, ataxia, and areflexia consistent with MF; however he had limb weakness, a common symptom presented in classic GBS [5, 16]. Due to the overlap of neurologic signs, the final diagnosis was MF/GBS. In Puerto Rico, the most common clinical symptoms documented in patients with GBS and ZIKV infection includes areflexia, leg weakness, leg paresthesia, arm weakness, facial weakness, arm numbness, and dysphagia. Among cases with neurologic signs due to GBS, nearly all of cases reported areflexia (97\%), while $63 \%$ of patients had facial weakness. Most of them were admitted to ICU $(63 \%)$, while $35 \%$ required mechanical ventilation [7].

GBS generally has favorable outcomes; however a poor prognosis has been associated with rapid onset and severity of weakness [17]. In the current case, severe headache was described as one of the initial neurologic signs, and 48 hours after the initial dose of IVIg treatment. Back and extremities 
pain is frequent in GBS, however headache is a rare clinical manifestation barely reported but presented in some variants of GBS, mainly MF and MF/GBS overlap syndrome [18, 19]. The pathogenesis of headache has been attributed to inflammation of the trigeminovascular pathway and increased of cerebrospinal fluid protein leading to obstruction and intracranial pressure [19, 20]. IVIg is considered a safe treatment, yet adverse reactions could vary between $1-80 \%$ of cases [21]. As in the current case, adverse reaction after the first 48 hours of treatment are considered delayed reactions attributed to exacerbating factors, including previous episodes of headaches [21]. In this case, electrophysiological findings and antiGQ1b antibodies results were not available; furthermore albuminocytologic dissociation and improvement upon IVIg treatment were consistent with the suspected diagnosis. Most of MFS cases confirmed with anti-GQ1b antibodies have ocular involvement with cranial nerves III, IV and VI; however in other cases with multiple cranial nerves as well as isolated ophthalmoplegia, anti-GQ1B antibodies has been identified [22]. As recorded, the patient requested medical care on multiples times. Healthcare resource needs during active ZIKV transmission on 2016 was 3-5 times greater as compared with non-active ZIKV transmission; [8] therefore, the quick response among healthcare providers is essential for the recovery process, to reduce long-term effects, and healthcare resources needed during the outbreak. Early treatment initiation, including plasma exchange or IVIg infusion therapy should be considered, particularly in patients presenting with a rapid progression of symptoms, including paralysis, weakness, and pain.

\section{References}

1. Dirlikov E, Ryff KR, Torres-Aponte J, et al. Update: Ongoing Zika virus transmissionPuerto Rico. MMWR Morb Mortal Wkly Rep 2016; 65(17):451-55.

2. Sharp TM, Muñoz-Jordán J, Perez-Padilla J, et al. Zika virus associated with severe thrombocytopenia. Clin Infect Dis 2016; 63(9):1198-1201.

\section{Conclusions}

ZIKV infection should be considered in patients presenting with the most common clinical symptoms of MF syndrome associated to the ZIKV infection, including residents and travelers from endemic areas. Due to DENV being endemic in Puerto Rico, in cases were serologic testing indicates DENV and/or ZIKV patients should be managed for both infections due to possible infection of either viruses.

\section{Abbreviations}

ZIKV: Zika Virus; GBS: Guillain-Barré syndrome; MF: Miller Fisher; ICU: Intensive Care Unit; MF/GBS: Miller Fisher-GuillainBarré overlap syndrome; ER: Emergency room; DENV: Dengue virus; ELISA: enzymelinked immunosorbent array; IVIg: Intravenous Immunoglubin

\section{Conflict of interest}

Gloria M. Rodríguez-Vega: Speaker Burial The Medicines Company-Cleviprex; Wilma GonzálezBarreto, Patricia De Jesús-Umpierre, and José R. Rodríguez-Vázquez declare no conflict of interests.

\section{Contribution}

All authors have contributed to this manuscript for: concept, design, drafting, and final revision.

\section{Acknowledgement}

Emilio Dirlikov, PhD - Puerto Rico Department of Health, San Juan, P.R., U.S.A \& Center of Disease Control and Prevention, Atlanta, Georgia, U.S.A.

\section{Informed Consent}

The Institutional Review Board of San Juan Bautista School of Medicine approved this study (EMSJB 92016). Consent was obtained from the patient for publication of this case report.

3. Cao-Lormeau V, Blake A, Mons $\mathrm{S}$, et al. Guillain-Barré syndrome outbreak associated with Zika virus infection in French Polynesia: a case-control study. Lancet 2016; 387(10027):1531-1539.

4. Hennessey M, Fischer M, Staples E. Zika virus spreads to new areas - region of the Americas, 
May 2015-January 2016. MMWR Morb Mortal Wkly Rep 2016; 65(3):55-58.

5. Yuki N, Hartung HP. Guillain-Barré syndrome. N Engl J Med 2012; 366(24):2294-2304.

6. Yepishin IV, Allison RZ, Kaminskas DA, Zagorski NM, Liow KK. Miller Fisher syndrome: A case report highlighting heterogeneity of clinical features and focused differential diagnosis. Hawaii J Med Public Health 2016; 75(7):196-199.

7. Dirlikov E, Major CE, Mayshack $M$, et al. Guillain-Barré syndrome during ongoing Zika Virus transmission - Puerto Rico, January 1July 31, 2016. MMWR Morb Mortal Wkly Rep 2016; 65(34):910-914.

8. Dirlikov E, Kniss K, Major C, et al. GuillainBarré syndrome and healthcare needs during Zika virus transmission, Puerto Rico, 2016. Emerg Infect Dis 2017; 23(1):134-136.

9. Salinas JL, Major CG, Pastula DM, et al. Incidence and clinical characteristics of Guillain-Barré syndrome before the introduction of Zika virus in Puerto Rico. $J$ Neurol Sci 2017. doi: 10.1016/j.jns.2017.04.006

10. dos Santos $T$, Rodriguez A, Almiron M, et al. Zika virus and Guillain-Barré syndrome-Case series from seven countries. $N$ Engl $J$ Med 2016; 375:1598-1601.

11. Pan American Health Organization. Guidelines for surveillance of Zika virus disease and its complications. Washington, D.C.: PAHO, 2016. [http://iris.paho.org/xmlui/bitstream/handle/1234 56789/28405/9789275118948 eng.pdf?sequence $=1$ \&isAllowed $=y$ available at 26.12.2017]

12. Adams L, Bello-Pagán $M$, Lozier $M$, et al. Update: Ongoing Zika virus transmission Puerto Rico. MMWR Morb Mortal Wkly Rep 2016; 65(30):774-779.

13. Rabe IB, Staples JE, Villanueva J, et al. Interim guidance for interpretation of Zika virus antibody test results. MMWR Morb Mortal Wkly Rep 2016; 65.

14. Guidance for U.S. Laboratory testing for Zika virus infection: Implications for health care providers. MMWR Morb Mortal Wkly Rep 2016; 65(46):1304.

15. Sever M, Aksay E, Gulec F. The diagnosis is made only with suspicion: Miller Fisher syndrome. Hong Kong Jour Emerg Med 2011; 18(6):428-431.

16. Wakerley BR, Yuki N. Mimics and chamaleons in Guillain-Barré and Miller Fisher syndromes. Pract Neurol 2015; 15(2):90-99.

17. Sejvar JJ, Hohl KS, Gidudu J, et al. GuillainBarré syndrome and Fisher syndrome: Case definitions and guidelines for collection, analysis, and presentation of immunization safety data. Vaccine 2011; 29:599-612.

18. Farmakidis $C$, Inan S, Milstein M, Herskovitz S. Headache and pain in Guillain-Barré syndrome. Curr Pain Headache Rep 2015; 19:40.

19. Pirotta T, Chircop C, Vella N. Is headache a common symptom in Miller Fisher syndrome? two case reports. J Autoimmun Res 2016; 3(1):1011.

20. Friedman DI, Potts E. Headache associated with Miller Fisher syndrome. Headache 2007; 1347-1348.

21. Cherin $P$, Marie $I$, Michallet $M$, et al. Management of adverse events in the treatment of patients with immunoglubin therapy: A review of evidence. Autoimmun Rev 2015; 15(2016):71-81.

22. Odaka M, Yuki N, Hirata K. Anti-GQ1b lgG antibody syndrome: clinical and immunological range. J Neurol Neurosurg Psychiatry 2001; 70:50-55. 